ARTICLE

\title{
Protection from septic peritonitis by rapid neutrophil recruitment through omental high endothelial venules
}

\author{
Konrad Buscher 1,2,3, Huiyu Wang ${ }^{1,2}$, Xueli Zhang1,2, Paul Striewski ${ }^{2,4}$, Benedikt Wirth ${ }^{2,4}$, Gurpanna Saggu ${ }^{5}$, \\ Stefan Lütke-Enking ${ }^{1,2}$, Tanya N. Mayadas ${ }^{5}$, Klaus Ley ${ }^{6}$, Lydia Sorokin ${ }^{1,2}$ \& Jian Song ${ }^{1,2}$
}

Acute peritonitis is a frequent medical condition that can trigger severe sepsis as a lifethreatening complication. Neutrophils are first-responders in infection but recruitment mechanisms to the abdominal cavity remain poorly defined. Here, we demonstrate that high endothelial venules (HEVs) of the greater omentum constitute a main entry pathway in TNF $\alpha-$, Escherichia coli (E. coli)- and caecal ligation and puncture-induced models of inflammation. Neutrophil transmigration across HEVs is faster than across conventional postcapillary venules and requires a unique set of adhesion receptors including peripheral node addressin, E-, L-selectin and Mac-1 but not P-selectin or LFA-1. Omental milky spots readily concentrate intra-abdominal $\mathrm{E}$. coli where macrophages and recruited neutrophils collaborate in phagocytosis and killing. Inhibition of the omental neutrophil response exacerbates septic progression of peritonitis. This data identifies HEVs as a clinically relevant vascular recruitment site for neutrophils in acute peritonitis that is indispensable for host defence against early systemic bacterial spread and sepsis.

\footnotetext{
${ }^{1}$ Institute of Physiological Chemistry and Pathobiochemistry, University of Muenster, Muenster 48149, Germany. ${ }^{2}$ Cells-in-Motion Cluster of Excellence, University of Muenster, Muenster 48149, Germany. ${ }^{3}$ Department of Nephrology and Rheumatology, University of Muenster, Muenster 48149, Germany. ${ }^{4}$ Institute for Computational and Applied Mathematics, University of Muenster, Muenster 48149, Germany. ${ }^{5}$ Center for Excellence in Vascular Biology, Department of Pathology, Brigham and Women's Hospital and Harvard Medical School, Boston, Massachusetts 02115, USA. ${ }^{6}$ Division of Inflammation Biology, La Jolla Institute for Allergy and Immunology, La Jolla, California 92037, USA. Correspondence and requests for materials should be addressed to K.B. (email: kbuscher@uni-muenster.de) or to J.S. (email: songj@uni-muenster.de).
} 
M ultiple clinical conditions such as trauma, surgery, liver cirrhosis, peritoneal dialysis catheters or disruption of the gastrointestinal tract can cause peritonitis. Subsequent systemic spread of intra-abdominal pathogens results in sepsis with high mortality and medical treatment is challenging ${ }^{1}$.

Leucocyte influx into the peritoneal cavity is a hallmark of abdominal inflammation but the routes of neutrophil emigration remain enigmatic. Here we studied the greater omentum, an intraperitoneal organ deriving from the greater curvature of the stomach and the spleen. It consists of a double layer of mesothelial cells that encapsulates a highly vascularized parenchyma of fatty tissue and multiple immune cell aggregates called milky spots. These are considered unconventional secondary lymphoid organs ${ }^{2}$ and consist mostly of B1-cells, macrophages, T cells and mast cells, and lack follicular dendritic cells and germinal centres ${ }^{3,4}$. Milky spots dynamically increase in number and size in chronic inflammation or irritation ${ }^{5,6}$ and a role in natural antibody production and adaptive peritoneal immunity is well established ${ }^{2,7,8}$. A key feature is the presence of a specialized type of postcapillary venule called high endothelial venule $(\mathrm{HEV})$ that is essential for lymphocyte trafficking 9 . Similar innate B1-cell-rich clusters in adipose tissues have been observed in the mouse mesentery, the pleural cavity and the pericardium, and are termed fat-associated lymphoid clusters ${ }^{10,11}$.

To date, little is known about the regulatory functions of the greater omentum in innate immunity. Therefore, we studied its impact on leucocyte recruitment to and bacterial spread from the abdominal cavity in murine models of acute peritonitis. We can show that the first wave of neutrophils recruited to the inflamed peritoneum uses milky spot HEVs as exit site with unique molecular properties and that specific inhibition of this pathway leads to systemic bacterial spread and clinical exacerbation of septic peritonitis.

\section{Results}

Omental HEVs as neutrophil exit route in peritonitis. Milky spots are located predominantly at the lateral border of the greater omentum and peripheral node addressin (PNAd) as key marker for HEVs can be detected using the MECA-79 monoclonal antibody (Fig. 1a; Supplementary Fig. 1a) ${ }^{9}$. At steady state, there are few neutrophils present in the omentum or the abdominal cavity, but neutrophil influx can be provoked by intraperitoneal application of TNF $\alpha$ (Fig. 1b) ${ }^{12}$. Omentectomized mice showed a strong reduction in peritoneal neutrophil influx at $1 \mathrm{~h}$ after TNF $\alpha$ application indicating a functional role of the greater omentum in acute peritonitis (Fig. 1b). Using $L y s M-e G F P$ reporter mice in which neutrophils are brightly fluorescent ${ }^{13}$ with $1 \mathrm{~h}$ intraperitoneal (i.p.) TNF $\alpha$ treatment, we observe that neutrophil invasion is first triggered in milky spots in close vicinity to HEVs (Fig. 1c). Electron microscopy revealed polymorphonuclear cells in close contact to lymphocytes at luminal, subendothelial and perivascular sites in inflamed milky spots (Fig. 1d) suggesting a similar exit site for both leucocyte subsets.

Next, we developed a surgical technique to perform intravital microscopy on the omental microcirculation (Supplementary Fig. 1b) showing early neutrophil-endothelium interactions in milky spots and parenchymal neutrophil migration adjacent to HEVs in LysM-eGFP mice (Supplementary Movies 1 and 2; Supplementary Fig. 1c). Intravital 4D spinning disc confocal microcopy confirmed HEVs as predilection site for active neutrophil transmigration in acute TNF $\alpha$-induced inflammation (Fig. 1e, Supplementary Movie 3). A similar phenotype was observed in a neutrophil-specific Ly6G-tdTomato reporter mouse $^{14}$ (Supplementary Movie 4).
We noticed that at early $(1 \mathrm{~h})$ but not later stages $(>3 \mathrm{~h})$ of TNF $\alpha$-inflammation neutrophils accumulated intraluminally in PNAd ${ }^{-} /$PECAM- ${ }^{+}$postcapillary venules (PCV; Fig. 1c; Supplementary Fig. 2a). Tracking of neutrophil motion $60 \mathrm{~min}$ after TNF $\alpha$ revealed distinct HEV exit ramps as described for lymphocytes ${ }^{15}$, whereas PCVs predominantly showed rolling neutrophils (Fig. 1f). Time-lapse video recordings indicate a different temporospatial response of neutrophil recruitment in HEVs compared with PCVs with the former being dominant in the first $60-80 \mathrm{~min}$ of TNF $\alpha$-inflammation (Fig. 1g; Supplementary Fig. 2b; Supplementary Movie 5). The first wave of neutrophil egress out of the omental parenchyma into the surrounding area is initiated in close vicinity to milky spot HEVs as shown by intravital microscopy (Fig. 1h). Together, this data indicates that HEVs constitute a neutrophil exit platform in the early phase of peritoneal inflammation allowing rapid neutrophil entry into omental milky spots and subsequent release into the abdominal cavity.

Unique requirement of adhesion molecules. To delineate the molecular pathway of HEV-mediated neutrophil recruitment, we blocked adhesion molecules by monoclonal antibodies. Flow cytometry showed a population of $\mathrm{CD} 11 \mathrm{~b}^{+} \mathrm{Ly}_{6 \mathrm{G}}{ }^{+}$neutrophils in the abdominal cavity already within $30 \mathrm{~min}$ and a further increase at $60 \mathrm{~min}$ after TNF $\alpha$ (Fig. 2a). PNAd blockade by $50 \mu \mathrm{g}$ MECA-79 mAb abolished most neutrophil influx, but did not affect $\mathrm{CD} 11 \mathrm{~b}^{+} \mathrm{Ly6G}^{-}$macrophages (Fig. 2a). Likewise, we found an absolute requirement for L-selectin (CD62L), E-selectin (CD62E), P-selectin glycoprotein ligand-1 (PSGL-1), CD11b/ CD18 (Mac-1) and intercellular adhesion molecule 1 (ICAM-1), whereas P-selectin $(\mathrm{CD} 62 \mathrm{P}), \alpha_{4} \beta_{7}$-integrin and $\mathrm{CD} 11 \mathrm{a} / \mathrm{CD} 18$ (LFA-1) did not play a significant role as assessed at the $60 \mathrm{~min}$ time point (Fig. 2b). A similar effect of blocking antibodies on neutrophil recruitment was determined in omental extracts (Supplementary Fig. 3a) and in response to intraperitoneal bacterial lipopolysaccharide (Supplementary Fig. 3b). We confirmed the unexpected role of Mac-1 using knockout mice ${ }^{16}$ showing reduced neutrophil numbers in TNF $\alpha$-inflamed omenta compared with wild-type controls (Supplementary Fig. 3c).

Intravital imaging indicated that intraluminal neutrophils exhibited rolling, crawling and arrest in inflamed HEVs (Fig. 2c). Blocking concentrations of MECA-79 mAb abolished most neutrophil-HEV interactions (Fig. 2c; Supplementary Movie 6). E-selectin can be detected in TNF $\alpha$-exposed HEVs using immunofluorescence microscopy (Supplementary Fig. 3d) and its inhibition induced a prominent crawling phenotype (Fig. 2c; Supplementary Movie 7). Mac-1 blockade prevented arrest and crawling, leaving only rolling neutrophils (Fig. 2c; Supplementary Movie 8).

Inhibition of HEV-mediated neutrophil exit aggravates sepsis. Our data suggested that the greater omentum may play a role in the early innate immune response of bacterial infections. We used a sub-lethal caecal ligation and puncture (CLP) model to induce polymicrobial peritonitis and found that substantial neutrophil recruitment in omental milky spots is triggered at $4 \mathrm{~h}$ after surgery, whereas sham-operated controls showed only little signs of inflammation (Fig. 3a and Supplementary Fig. 4a). To investigate the spread of abdominal bacteria, fluorescently labelled E. coli were injected i.p. Confocal imaging of the omentum after $2 \mathrm{~h}$ incubation revealed their presence predominantly in milky spots (Fig. 3b). Recruited neutrophils could be observed in close vicinity to milky spot HEVs and largely colocalize with E. coli (Fig. 3b, Supplementary Movie 9). Flow cytometry showed that $\mathrm{CD} 11 \mathrm{~b}^{+} \mathrm{F} 4 / 80^{+}$-macrophages but also $\mathrm{CD} 11 \mathrm{~b}^{+} \mathrm{Ly}_{6 \mathrm{G}}{ }^{+}$ 
a

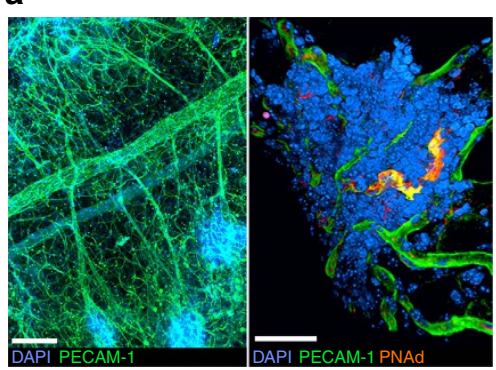

b

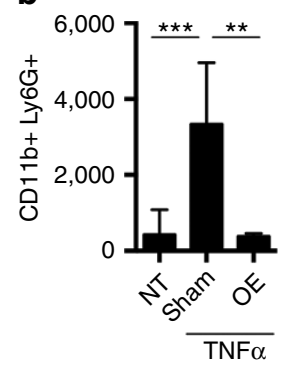

c

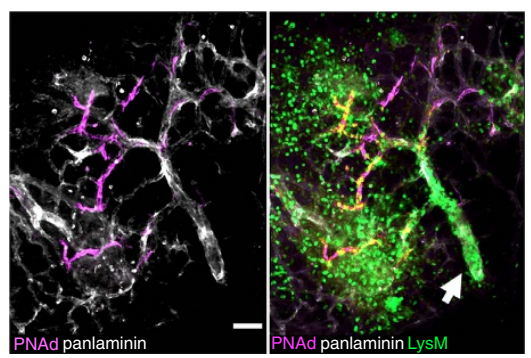

d

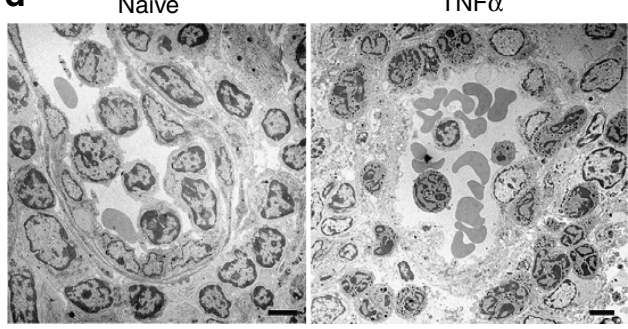

e

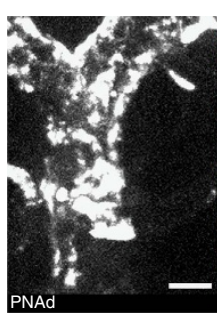

$t=5 \min$

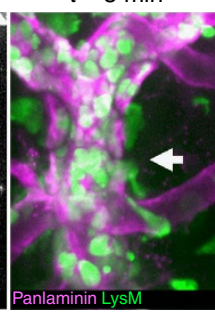

$t=8 \mathrm{~min}$

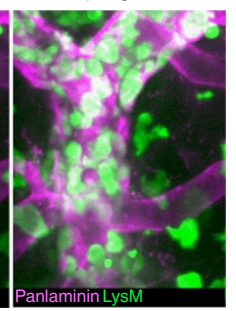

f

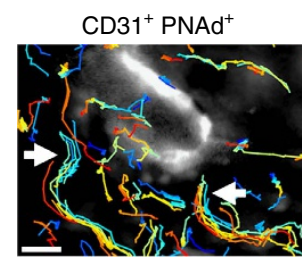

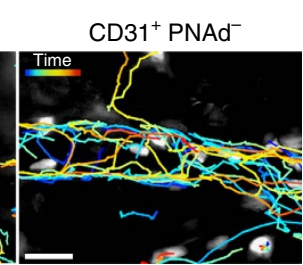

g

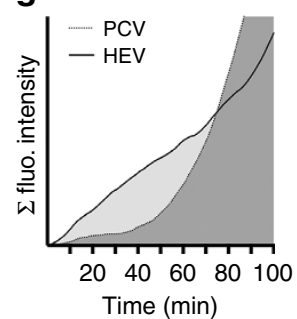

h

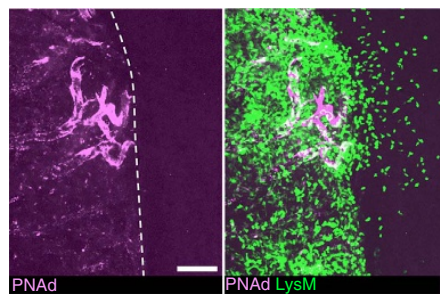

Figure 1 | HEVs of the greater omentum enable rapid neutrophil exit in TNF $\alpha$-peritonitis. (a) Confocal micrographs of the naive omentum show vascularization and milky spots (left panel). PNAd ${ }^{+}$PECAM-1+- HEVs are distinct vascular compartments within the milky spot (right panel). Scale bars, $120 \mu \mathrm{m}$ (left) and $40 \mu \mathrm{m}$ (right). (b) Non-treated (NT), omentectomized (OE) or sham-operated mice were compared by analysing the peritoneal lavage after 60 min i.p. treatment with TNF $\alpha$. Cell number as mean \pm s.d. $n=5$ per condition of three independent experiments. Student's $t$-test ${ }^{\star \star} P<0.01$, ${ }^{\star \star \star} P<0.001$. (c) Confocal micrograph of an inflamed omentum of a LysM-eGFP reporter mouse after 60 min i.p. TNF $\alpha$ treatment. Panlaminin is used as a vessel marker. Note the luminal accumulation of GFPbright neutrophils in the PNAd ${ }^{-}$-postcapillary venule (arrow). Scale bar, $40 \mu \mathrm{m}$. Representative of five independent experiments. (d) Transmission electron micrographs of naive and TNF $\alpha$-inflamed milky spot HEVs. Scale bar, $5 \mu \mathrm{m}$. Representative of $>10$ milky spots in two independent experiments. (e) Representative snapshots from a 4D intravital spinning disc microscopy video with PNAd- and panlaminin staining using a LysM-eGFP mouse (video 3). The arrow indicates extravasating GFPbright neutrophils. Scale bar, $20 \mu \mathrm{m}$. (f) Video recordings of PNAd ${ }^{+}$ HEVs and PNAd ${ }^{-}$PCV were used for LysM-eGFP neutrophil tracking 60 min after TNF $\alpha$ application. Colour-coding of tracks indicates time (20 min). HEV stained by MECA-79 in white. Arrows indicate active neutrophil transmigration sites. Representative of three independent experiments. Scale bar, $20 \mu \mathrm{m}$. (g) Analysis of an intravital recording of a milky spot HEV and a conventional PCV in the same field of view over a time course of 100 min in a LysM-eGFP mouse. TNF $\alpha$ was topically applied at $0 \mathrm{~min}$ and the integral of the pixel intensity as a measure of leucocyte accumulation in the region of interest was plotted over time. Data representative of four milky spots in two independent experiments. (h) Intravital microscopy of the inflamed exteriorized omentum in LysM-eGFP mice with PNAd staining. The dashed line marks the omental border. Scale bar, $100 \mu \mathrm{m}$. Representative of two independent experiments.

neutrophils can phagocytose bacteria (Fig. 3c). Blocking L-selectin or PNAd abrogates neutrophil phagocytosis whereas macrophage function, assessed at $2 \mathrm{~h}$ after E. coli injection, is unaffected in both peritoneal lavage (Fig. 3c) and in omentum extracts (Supplementary Fig. 4c). Next, we injected a sub-lethal dose of E. coli i.p. and assessed sepsis using a murine sepsis score ${ }^{17}$. While control mice developed mild clinical signs over a 2-h time course, blocking PNAd or L-selectin disabled the protective neutrophil response (Supplementary Fig. 4d) which was associated with pronounced sepsis starting $\sim 45 \mathrm{~min}$ after E. coli injection (Fig. 3d). The severe clinical phenotype was associated with greatly increased bacterial loads in the omentum, peritoneal lavage and blood (Fig. 3e). Bacteremia was increased 50 to -100 fold in L-selectin blocked mice. The clinical sepsis score was positively correlated with bacterial burden of the omentum (Fig. 3f). This data suggests neutrophil recruitment via omental milky spot HEVs to be critical for abdominal pathogen clearance and preventing early onset of sepsis.

\section{Discussion}

The outstanding capability of the greater omentum to resolve inflammation and fight bacteria has been clinically appreciated for many decades but the underlying pathomechanisms remain mostly unknown ${ }^{18}$. We report here that a hyperacute phase of mono- and polymicrobial peritoneal inflammation induces rapid neutrophil influx into the omentum by means of milky spot HEVs.

The spatial and temporal molecular pattern of leucocyte- and endothelial-expressed adhesion molecules, chemokines and other regulatory proteins defines the quality and quantity of local cell recruitment. Organ-specific variations of the adhesion cascade 
a

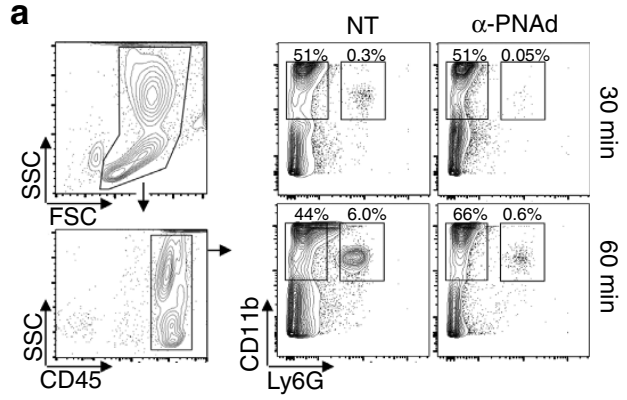

b

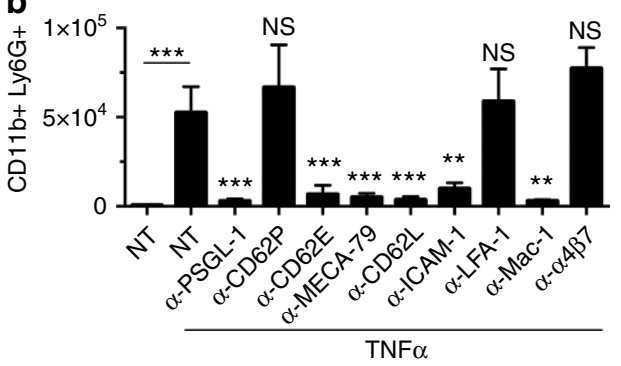

C

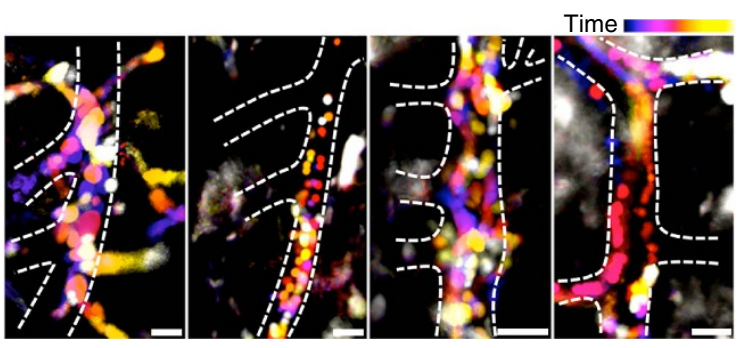

NT

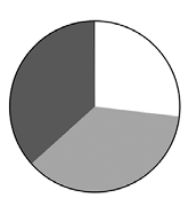

$\alpha-P N A d$

$\alpha$-E-selectin

$\alpha-M a c-1$
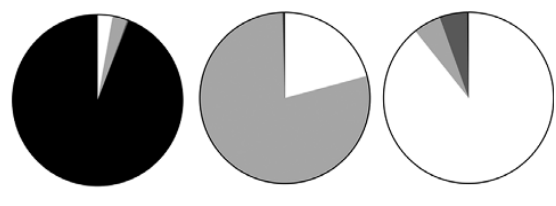

$\square$ Crawling

$\square$ Arrest

No interaction

Figure 2 | Unique adhesion receptor requirements of HEV-mediated neutrophil recruitment. (a) Flow cytometry gating strategy and analysis of the peritoneal lavage after 30 or 60 min of TNF $\alpha$-induced peritonitis with and without (NT, non-treated) PNAd inhibition by high-dose i.v. MECA-79 mAb injection. Data representative of two independent experiments. (b) The effect of different blocking antibodies on neutrophil accumulation in the abdominal lavage was evaluated after $1 \mathrm{~h} \mathrm{TNF} \alpha$ treatmensing flow cytometry as in (a). $n=6$ of least three independent experiments. Data as mean total cell number \pm s.d. Statistical significance is indicated relative to NT with TNF $\alpha$. Student's $t$-test ${ }^{\star \star} P<0.01,{ }^{\star \star \star} P<0.001$. (c) To investigate the luminal neutrophil behaviour, E-selectin, L-selectin or Mac-1 was blocked by i.v. injection of monoclonal antibodies and intravital microscopy of inflamed omental milky spots was performed in LysM-eGFP mice at high magnification. Time is colour-coded and visualizes cell motion within 3-5 min duration. Dashed lines indicate vessel borders. Scale bar, $20 \mu \mathrm{m}$. Quantification of intraluminal neutrophil behaviour in HEVs shown as pie charts for each condition. Cell numbers for rolling/crawling/arrest/time without any neutrophil-endothelium interaction in seconds: NT $n=25 / 33 / 34 / 0, \alpha$-PNAd $=1 / 1 / 0 / 210, \alpha$-E-selectin $n=9 / 34 / 0 / 0$ and $\alpha-M a c-1 n=34 / 2 / 2 / 0$.

such as in liver, brain and lung have been described ${ }^{19,20}$. Several lines of evidence argue for a distinct mechanism of neutrophil recruitment in HEVs. We detected a shorter response time compared with PCV after TNF $\alpha$ stimulation. In fact, the mode of leucocyte diapedesis in HEVs seems to be unique as shown by a mouse model with locked interendothelial junctions ${ }^{21}$. Electron microscopy studies highlighted a discontinuous basement membrane and loose lining of mesothelial cells at milky spots that might facilitate fast egress into the peritoneal cavity 22,23 .

Furthermore, the panel of required adhesion receptors for neutrophil extravasation is exceptional. Constitutive lymphocyte homing to secondary lymphatic tissue requires L-selectin/PNAd and LFA-1/ICAM-1 interactions in HEVs for rolling and arrest, respectively ${ }^{9,24}$. It is known that neutrophils are also capable of rolling in HEVs at steady state in an L-selectin/PNAd-dependent manner ${ }^{24}$ and we show that this is a prerequisite in inflammatory conditions. These findings confirm previous studies on the impact of L-selectin ${ }^{25}$ and its ligand epitope 6-sulfo sialyl Lewis X (ref. 26) in abdominal inflammation and now identify the molecular and cellular mechanisms. In contrast to lymphocytes, Mac-1 and E-selectin but not LFA-1 critically contribute to neutrophil extravasation. Mac-1 is important for luminal crawling ${ }^{27}$ and blockade of E-selectin or its counterreceptor PSGL-1 fully abrogates the downstream adhesion cascade. Interestingly, intravital microscopy reveals that the transition from crawling to arrest requires E-selectin engagement. This finding corroborates a recent report on constitutive E-selectin transcription in HEVs (ref. 28) suggesting modulatory functions in inflammation. While inside-out signalling to LFA-1 is well documented $^{29,30}$, a role of E-selectin in LFA-1-independent arrest remains to be investigated.
A rapid phagocyte response is decisive for successful resolution of infection ${ }^{31,32}$. Our study attributes the greater omentum a critical task of particularly rapid neutrophil deployment in the peritoneal cavity via HEV-specific mechanisms. Inhibition of this pathway increases bacterial load in the peritoneum and exacerbates septic peritonitis. The critical time window is also known from clinical settings and referred to as 'golden hours of sepsis $^{33}$. Some data hint at an association between omentectomy and higher sepsis rates and mortality in patient ${ }^{34,35}$. However, murine peritonitis models do not seem to fully recapitulate human pathophysiology ${ }^{36}$ and clinical studies are needed to definitively address the role of the greater omentum in inflammation and sepsis in humans.

The novel insight of HEVs as a distinct vascular entity for neutrophil exit may be relevant in a number of inflammatory and malignant disorders with formation of tertiary lymphoid organs ${ }^{37}$. In accordance with our findings, neutrophil infiltration around lymph node HEVs with E-selectin expression has been described in conditions with elevated TNF $\alpha$ levels such as granulomatosis and Hodgkin's disease ${ }^{28,38,39}$. The fact that HEV-mediated neutrophil recruitment relies on a unique pattern of adhesion receptors could possibly pave the way for new therapeutic strategies to modulate inflammation.

\section{Methods}

Animals. C57BL/6J (Jackson Laboratories), LysM-eGFP (ref. 13; kindly provided by D. Vestweber), Mac-1 knockout ${ }^{16}$ and Ly6G-tdTomato mice ${ }^{14}$ (kindly provided by $\mathrm{M}$. Gunzer; all transgenic strains on C57BL/6J background) of both genders were used at the age between 8 and 30 weeks. Animal breeding and experiments were conducted according to the German Animal Welfare guidelines and were approved by the Animal Ethics Committee of the University of Muenster. E. coli and CLP peritonitis experiments were performed at the La Jolla Institute for 
a

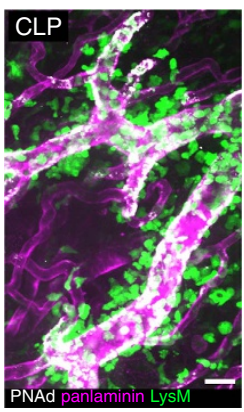

b

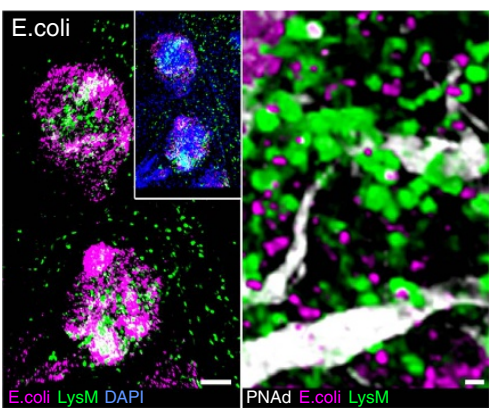

C

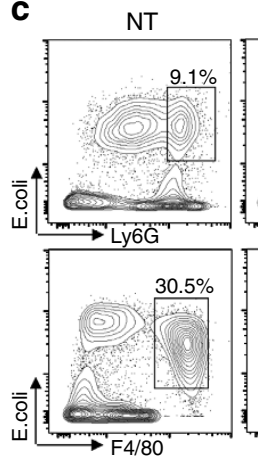

$\alpha-C D 62 L$

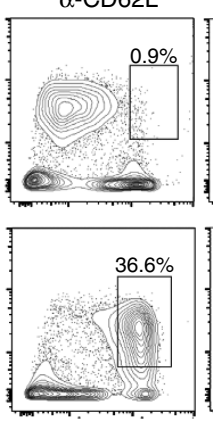

$\alpha-$ PNAd

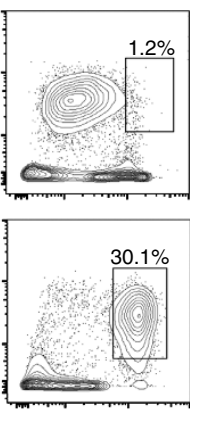

d

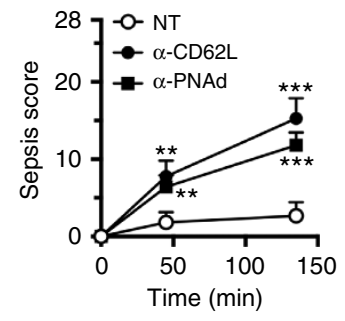

e

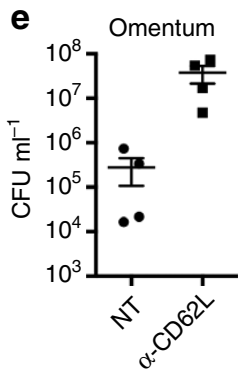

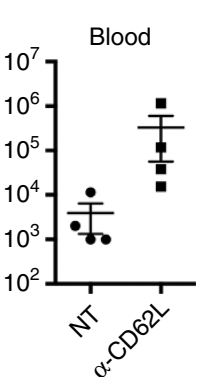

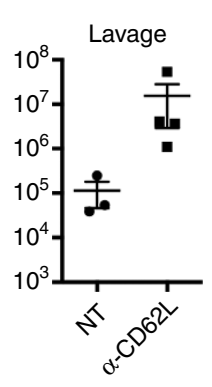

f

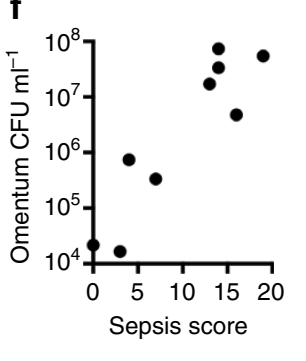

Figure 3 | Bacterial peritonitis triggers HEV-mediated neutrophil recruitment required to prevent septic peritonitis. (a) Polymicrobial sepsis was induced by caecal ligation and puncture and the greater omentum was imaged by confocal microscopy $4 \mathrm{~h}$ after surgery. Sham control did not show major signs of inflammation (Supplementary Fig. 4a). Representative of three independent experiments. Scale bar, $30 \mu \mathrm{m}$. (b) Fluorescently labelled E. coli were injected i.p. and confocal micrographs of the omentum were taken after $2 \mathrm{~h}$. The left panel shows a low magnification image of two milky spots. The right panel shows LysM-eGFP neutrophils adjacent to HEVs and labelled E. coli. Scale bars, $100 \mu \mathrm{m}$ (left) and $10 \mu \mathrm{m}$ (right). (c) Flow cytometry data of the peritoneal lavage showing colocalization of fluorescently labelled E. coli and CD11b + F4/80 ${ }^{+}$macrophages or CD11b ${ }^{+}$Ly6G ${ }^{+}$neutrophils $2 \mathrm{~h}$ after infection in untreated (NT, non-treated), L-selectin- or PNAd-blocked mice. Similar data has been obtained for omental extracts (Supplementary Fig. 4c). Data is representative of two independent experiments. Gating strategy on CD $45^{+}$CD11b ${ }^{+}$cells in Supplementary Fig. $4 \mathrm{~b}$. (d) Clinical evaluation of mice i.p. injected with live E. coli using a murine sepsis score with a maximum of 28 points ${ }^{17}$. PBS-treated $(N T, n=6)$, blocking anti-L-selectin antibody $(n=4)$ or blocking anti-PNAd antibody treated $(n=5)$ mice were compared over a 2-h time course in 2-3 independent experiments. Data shows mean \pm s.d. Significance shown compared with untreated condition. Two-way analysis of variance with Bonferroni posthoc correction. ${ }^{\star \star} P<0.01$, ${ }^{\star \star \star} P<0.001$. (e) The abdominal lavage, the greater omentum and blood was collected after $2 \mathrm{~h}$ E. coli peritonitis for the determination of colony forming units (CFUs). Data shows mean \pm s.d. (f) Correlation of omental E. coli bacterial load and the maximal clinical sepsis score obtained during $2 \mathrm{~h}$ after infection. Each data point represents one mouse of the untreated or anti-L-selectin treated groups at the $120 \mathrm{~min}$ time point.

Allergy and Immunology with the approval of the institutional Animal Care Committee. Peritonitis experiments on Mac-1 knockout mice were performed in the Department of Pathology, Brigham and Women's Hospital.

Reagents. The following anti-mouse monoclonal antibodies were purchased from BD Bioscience: anti-L-selectin (clone MEL-14, \#553147), anti-PSGL-1 (clone 4RA10, \#557787), anti-CD11b (clone M1/70, \#553308), anti-E-selectin (clone 10E9.6, \#550290) and anti-P-selectin (clone RB40.34, \#553741). Anti-CD11a (clone 17.4, \#22850110) and anti-ICAM-1 (clone YNI.7.4, \#22270540) were purchased from Immunotools. Anti-Integrin $\beta 7$ (clone FIB504, \#14-5867-82) was bought from eBioscience. A polyclonal antibody against mouse panlaminin was purchased from Sigma Aldrich (\#L9393). Recombinant mouse TNF $\alpha$, anti-mouse MECA-79 (2-3 $\mu \mathrm{g}$ for labelling, $20 \mu \mathrm{g}$ for blocking) and PECAM-1 (10 $\mu \mathrm{g}$ for labelling) antibodies were purified from hybridomas (provided by V. Wixler, E. Butcher and D. Vestweber, respectively). Antibody were labelled using amine-reactive fluorescent DyLight 488, 550 and 650 kits (Thermo Fisher). For confocal imaging, the secondary antibodies goat anti-rabbit DyLight 649 (Dianova, $2 \mu \mathrm{g} \mathrm{ml}^{-1}$ ) and donkey anti-rat Alexa Fluor 555 (Abcam, $2 \mu \mathrm{g} \mathrm{ml}^{-1}$ ) were used.

Peritonitis models. Acute peritonitis was induced by i.p. injection of TNF $\alpha$ $(500 \mathrm{ng})$ in $500 \mu \mathrm{l}$ PBS. In some experiments, blocking antibodies $(40 \mu \mathrm{g}$ in $100 \mu \mathrm{l}$ PBS) were administered intravenous (i.v.) $15 \mathrm{~min}$ before. After 30,60 or $120 \mathrm{~min}$ animals were euthanized by $\mathrm{CO}_{2}$ and the abdominal cavity was flushed with $5 \mathrm{ml}$ cold PBS. Subsequently, the omentum was extracted for further analysis. Alternatively, LPS (25 $\mu \mathrm{g}$, Sigma) was used with an incubation time of $12 \mathrm{~h}$.

The CLP model was performed according to a published protocol with a sub-lethal mortality ${ }^{40}$. We confirmed the low-mortality time course of this protocol (Supplementary Fig. 4e). Briefly, anaesthesia was induced using isoflurane and buprenorphine was administered subcutaneously for post-operative analgesia.
After a 1-cm midline incision of the abdomen the caecum was exteriorized. At $1 \mathrm{~cm}$ from the distal end, the caecum was ligated using 2-0 suture and perforated using a 27 gauge needle (both sides of the caecum). For short time points $(<4 \mathrm{~h})$, the animal was kept in anaesthesia after the operation until euthanasia and subsequent analysis. Sham operation as control did not include ligation and perforation.

For live E. coli experiments, non-pathogenic DH5 $\alpha$ were cultured in lysogeny broth medium overnight. Before each experiment, E. coli were incubated for $2 \mathrm{~h}$ in fresh medium to induce log phase. After i.v. administration of blocking antibody or vehicle, $1 \times 10^{8}$ E. coli were injected i.p. in $300 \mu \mathrm{l}$ PBS and clinical signs of sepsis were assessed using a murine sepsis score with a maximum of 28 points ${ }^{17}$. After $2 \mathrm{~h}$ the peritoneal lavage, the omentum and blood (in EDTA) was obtained and plated on lysogeny broth agar plates in serial dilutions. Following $24 \mathrm{~h}$ of incubation at $37^{\circ} \mathrm{C}$, the number of colonies was determined and expressed as mean of the diluted series.

For imaging studies, heat-inactivated fluorescently labelled E. coli (Bioparticles) were purchased from Molecular Probes. Particles $\left(6 \times 10^{7}\right)$ were diluted in $500 \mu \mathrm{l}$ PBS and injected i.p. After $2 \mathrm{~h}$, peritoneal lavage and omentum were harvested for confocal microscopy or flow cytometry analysis.

Intravital microscopy. Mice were injected with fluorescently labelled antibodies i.v. and anaesthetized by i.p. injection of ketamine $\left(125 \mathrm{mg} \mathrm{kg}^{-1}\right)$ and xylazine $\left(12.5 \mathrm{mg} \mathrm{kg}^{-1}\right)$. In some experiments a carotid catheter was inserted to readminister anaesthesia, inject antibodies or take blood samples. All antibodies used in vivo were azide-free. After removal of abdominal fur, a $1-1.5-\mathrm{cm}$ median incision was done at the linea alba of the upper abdomen to exteriorize the greater omentum. It extends from the greater curvature of the stomach and the spleen and can be distinguished from mesenterial fatty tissue by its lighter appearance. Gentle handling with cotton-wool tips is essential to avoid tissue damage and bleeding was electrocautered if necessary. A custom-made microscopic stage allowed fixation of the omentum between two cover slips. Throughout the experiment the animals 
were kept warm on a $37^{\circ} \mathrm{C}$ heating pad or incubation chamber and the exteriorized specimen was kept moist using heated bicarbonate-buffered saline. Omental inflammation was induced by topically applying TNF $\alpha\left(2.0 \mu \mathrm{g} \mathrm{ml}^{-1}\right)$ in PBS. To visualize HEVs only low doses of MECA-79 antibody ( $2-3 \mu \mathrm{g}$ per mouse) were injected. $200 \mathrm{kDa}$ FITC- or rhodamine-dextran was used as blood tracer. Videos were recorded using either a Axio Scope.A1 microscope (Zeiss) at $\times 10$ (numerical aperture 0.25 ) or $\times 20$ (numerical aperture 0.45 ) equipped with stroboscopic epifluorescent illumination (Colibri 2, Zeiss) or an upright spinning disc microscope (Zeiss Imager Axio Examiner D1). After imaging, mice were euthanized in anaesthesia and the omentum was extracted and fixed in $2 \%$ paraformaldehyde for further analysis.

Data analysis and cell tracking was done using $\mathrm{Fiji}^{41}$. Where indicated, movies were denoised via total variation denoising (according to ref. 42 with $\beta=0$ ). All video frames were rigidly aligned based on minimizing the so-called L1-distance between them. Colour channels with strong photobleaching but no other temporal change were replaced by the first frame to improve their visibility.

Omentectomy. Mice were anaesthetized as described above. A 1-cm incision was made in the upper linea alba to expose the greater curvature of the stomach. The omentum was clamped on both most lateral ends using micro clips $(0.8 \times 5 \mathrm{~mm})$, ligated and subsequently removed. Great care was taken to avoid bleeding. Next, $500 \mathrm{ng}$ TNF $\alpha$ in $500 \mu \mathrm{l}$ PBS were applied into the peritoneal cavity and the abdominal wall was closed up. After $1 \mathrm{~h}$, the abdominal cavity was lavaged with $2 \mathrm{ml}$ cold PBS. Sham operation consisted of abdominal incision and gentle manipulation of the omentum.

Flow cytometry. Extracted omenta were meshed using a $70-\mu \mathrm{m}$ strainer and washed using PBS. Cells were stained using standard flow cytometry protocols with a combination of anti-CD11b-FITC or -APC, anti-Ly6G-FITC or -APC, anti-F4/80-FITC or -APC and CD45-PerCP (1:200 dilution, all from BD Bioscience). Cell viability of omenta and lavage were determined using ghostdye stain (Tonbo) and was $>85 \%$.

Confocal microscopy. Omenta were isolated, fixed in $2 \%$ PFA for $1 \mathrm{~h}$, blocked with $5 \%$ foetal calf serum for $30 \mathrm{~min}$ and incubated with primary antibodies in PBS at $4{ }^{\circ} \mathrm{C}$. Specimens were subsequently stained with the secondary antibodies for $4 \mathrm{~h}$ at $4{ }^{\circ} \mathrm{C}$. Whole mount tissues were examined using a Zeiss LSM 700 confocal microscope equipped with a Hamamatsu ORCA-ER CCD camera. Images were analysed using Volocity software (ImproVision).

Electron microscopy. Milky spots were manually dissected from the omentum, fixed, dehydrated and embedded in epon resin using standard protocols. Sections $(60 \mathrm{~nm}$ thin) were cut on a microtome and stained with uranyl acetate and lead citrate. Images were taken using a Philips CM10 electron microscope. Milky spots were identified by the high density of parenchymal leukoytes. HEVs showed a higher cell morphology and were luminally populated with mononuclear cells.

Statistics. Data were analysed using Prism software (GraphPad). Unpaired Student's $t$-test or two-way analysis of variance with Bonferroni posthoc correction was used to compare conditions. Data are expressed as mean \pm s.d. ${ }^{*} P<0.05$, ${ }^{* *} P<0.01,{ }^{* *} P<0.001$

\section{References}

1. Sartelli, M. et al. Current concept of abdominal sepsis: WSES position paper. World J. Emerg. Surg. 9, 22 (2014).

2. Rangel-Moreno, J. et al. Omental milky spots develop in the absence of lymphoid tissue-inducer cells and support $\mathrm{B}$ and $\mathrm{T}$ cell responses to peritoneal antigens. Immunity 30, 731-743 (2009).

3. Krist, L. F. et al. Cellular composition of milky spots in the human greater omentum: an immunochemical and ultrastructural study. Anat. Rec. 241, 163-174 (1995).

4. Shimotsuma, M., Takahashi, T., Kawata, M. \& Dux, K. Cellular subsets of the milky spots in the human greater omentum. Cell Tissue Res. 264, 599-601 (1991).

5. Di Paolo, N. et al. Omental milky spots and peritoneal dialysis--review and personal experience. Perit. Dial. Int. 25, 48-57 (2005).

6. Van Vugt, E., Van Rijthoven, E. A., Kamperdijk, E. W. \& Beelen, R. H. Omental milky spots in the local immune response in the peritoneal cavity of rats. Anat. Rec. 244, 235-245 (1996).

7. Carlow, D. A., Gold, M. R. \& Ziltener, H. J. Lymphocytes in the peritoneum home to the omentum and are activated by resident dendritic cells. J. Immunol. 183, 1155-1165 (2009).

8. Ansel, K. M., Harris, R. B. S. \& Cyster, J. G. CXCL13 is required for B1 cell homing, natural antibody production, and body cavity immunity. Immunity 16, 67-76 (2002).
9. Girard, J.-P., Moussion, C. \& Förster, R. HEVs, lymphatics and homeostatic immune cell trafficking in lymph nodes. Nat. Rev. Immunol. 12, 762-773 (2012).

10. Moro, K. et al. Innate production of $\mathrm{T}(\mathrm{H}) 2$ cytokines by adipose tissueassociated c-Kit $(+)$ Sca-1 $(+)$ lymphoid cells. Nature 463, 540-544 (2010).

11. Bénézech, C. et al. Inflammation-induced formation of fat-associated lymphoid clusters. Nat. Immunol. 16, 819-828 (2015).

12. Song, J., Wu, C., Zhang, X. \& Sorokin, L. M. In vivo processing of CXCL5 (LIX) by matrix metalloproteinase (MMP)-2 and MMP-9 promotes early neutrophil recruitment in IL-1 $\beta$-induced peritonitis. J. Immunol. 190, 401-410 (2013).

13. Faust, N., Varas, F., Kelly, L. M., Heck, S. \& Graf, T. Insertion of enhanced green fluorescent protein into the lysozyme gene creates mice with green fluorescent granulocytes and macrophages. Blood 96, 719-726 (2000)

14. Hasenberg, A. et al. Catchup: a mouse model for imaging-based tracking and modulation of neutrophil granulocytes. Nat. Methods 12, 445-452 (2015).

15. Bajénoff, M. et al. Stromal cell networks regulate lymphocyte entry, migration, and territoriality in lymph nodes. Immunity 25, 989-1001 (2006).

16. Coxon, A. et al. A novel role for the beta 2 integrin CD11b/CD18 in neutrophil apoptosis: a homeostatic mechanism in inflammation. Immunity 5, 653-666 (1996).

17. Shrum, B. et al. A robust scoring system to evaluate sepsis severity in an animal model. BMC Res. Notes 7, 233 (2014).

18. Platell, C., Cooper, D., Papadimitriou, J. M. \& Hall, J. C. The omentum. World J. Gastroenterol. 6, 169-176 (2000).

19. Ley, K., Laudanna, C., Cybulsky, M. I. \& Nourshargh, S. Getting to the site of inflammation: the leukocyte adhesion cascade updated. Nat. Rev. Immunol. 7, 678-689 (2007).

20. Kolaczkowska, E. \& Kubes, P. Neutrophil recruitment and function in health and inflammation. Nat. Rev. Immunol. 13, 159-175 (2013).

21. Küppers, V., Vestweber, D. \& Schulte, D. Locking endothelial junctions blocks leukocyte extravasation, but not in all tissues. Tissue Barriers 1, e23805 (2013).

22. Hodel, C. Ultrastructural studies on the absorption of protein markers by the greater omentum. Eur. Surg. Res. 2, 435-449 (1970).

23. Doherty, N. S., Griffiths, R. J., Hakkinen, J. P., Scampoli, D. N. \& Milici, A. J. Post-capillary venules in the 'milky spots' of the greater omentum are the major site of plasma protein and leukocyte extravasation in rodent models of peritonitis. Inflamm. Res. 44, 169-177 (1995).

24. Von Andrian, U. H. Intravital microscopy of the peripheral lymph node microcirculation in mice. Microcirculation 3, 287-300 (1996).

25. Tedder, T. F., Steeber, D. A. \& Pizcueta, P. L-selectin-deficient mice have impaired leukocyte recruitment into inflammatory sites. J. Exp. Med. 181, 2259-2264 (1995).

26. Malý, P. et al. The $\alpha(1,3)$ fucosyltransferase fuc-TVII controls leukocyte trafficking through an essential role in L-, E-, and P-selectin ligand biosynthesis. Cell 86, 643-653 (1996).

27. Phillipson, M. et al. Intraluminal crawling of neutrophils to emigration sites: a molecularly distinct process from adhesion in the recruitment cascade. J. Exp. Med. 203, 2569-2575 (2006).

28. Lee, M. et al. Transcriptional programs of lymphoid tissue capillary and high endothelium reveal control mechanisms for lymphocyte homing. Nat. Immunol. 15, 982-995 (2014).

29. Zarbock, A., Lowell, C. A. \& Ley, K. Spleen tyrosine kinase Syk is necessary for E-selectin-induced alpha(L)beta(2) integrin-mediated rolling on intercellular adhesion molecule-1. Immunity 26, 773-783 (2007).

30. Lefort, C. T. \& Ley, K. Neutrophil arrest by LFA-1 activation. Front. Immunol. 3, 157 (2012)

31. Li, Y., Karlin, A., Loike, J. D. \& Silverstein, S. C. A critical concentration of neutrophils is required for effective bacterial killing in suspension. Proc. Natl Acad. Sci. USA 99, 8289-8294 (2002).

32. Malka, R., Wolach, B., Gavrieli, R., Shochat, E. \& Rom-Kedar, V. Evidence for bistable bacteria-neutrophil interaction and its clinical implications. J. Clin. Invest. 122, 3002-3011 (2012).

33. Sterling, S. A., Puskarich, M. A. \& Jones, A. E. Prehospital treatment of sepsis: what really makes the 'golden hour' golden? Crit. Care 18, 697 (2014).

34. Ambroze, W. L. et al. Let sleeping dogs lie: role of the omentum in the ileal pouch-anal anastomosis procedure. Dis. Colon Rectum 34, 563-565 (1991).

35. Traverso, L. W. \& MacFarlane, S. K. Pancreatic juice in the peritoneal cavity: antibiotics or omental preservation prevent mortality. J. Surg. Res. 43, 220-225 (1987).

36. Dejager, L., Pinheiro, I., Dejonckheere, E. \& Libert, C. Cecal ligation and puncture: the gold standard model for polymicrobial sepsis? Trends Microbiol 19, 198-208 (2011)

37. Aloisi, F. \& Pujol-Borrell, R. Lymphoid neogenesis in chronic inflammatory diseases. Nat. Rev. Immunol. 6, 205-217 (2006).

38. Ruco, L. P. et al. Cytokine production (IL-1 alpha, IL-1 beta, and TNF alpha) and endothelial cell activation (ELAM-1 and HLA-DR) in reactive 
lymphadenitis, Hodgkin's disease, and in non-Hodgkin's lymphomas. An immunocytochemical study. Am. J. Pathol. 137, 1163-1171 (1990).

39. Ruco, L. P. et al. Expression and cell distribution of the intercellular adhesion molecule, vascular cell adhesion molecule, endothelial leukocyte adhesion molecule, and endothelial cell adhesion molecule (CD31) in reactive human lymph nodes and in Hodgkin's disease. Am. J. Pathol. 140, 1337-1344 (1992).

40. Cuenca, A. G., Delano, M. J., Kelly-Scumpia, K. M., Moldawer, L. L. \& Efron, P. A. Current protocols in immunology: cecal ligation and puncture. Curr. Protoc. Immunol.. doi: 10.1002/0471142735.im1913s91 (2010).

41. Schindelin, J. et al. Fiji: an open-source platform for biological-image analysis. Nat. Methods 9, 676-682 (2012).

42. Bredies, K., Pock, T. \& Wirth, B. Convex relaxation of a class of vertex penalizing functionals. J. Math. Imaging Vis. 47, 278-302 (2013).

\section{Acknowledgements}

We are grateful to E. Butcher, D. Vestweber and V. Wixler for the MECA-79, PECAM-1 and TNF $\alpha$ hybridomas, respectively, and R. Hallmann for helpful discussions and suggestions. We thank U. Malkus and J. Klingauf for support with electron microscopy, and M. Gunzer and A. Hasenberg for providing Ly6G-tdTomato reporter mice. This work would not have been possible without funding from the German Research Foundation to L.S. (CRC1009/A02, So285/9-1, EXE1003), the Cells-in-Motion Cluster of Excellence Flexible Funds grant (FF-2014-07) to J.S., B.W. and K.B., a grant to K.B. from the Interdisciplinary Center for Clinical Research (IZKF) of the Medical Faculty of the University of Muenster (SEED 04/12) and a German Research Foundation grant to KB (BU 3247/1)

\section{Author contributions}

K.B. and J.S. performed experiments. J.S., K.B. and L.S. designed the study with the help of K.L. X.Z. and H.W. assisted with peritonitis experiments and S.L.E. with intravital microscopy. P.S. and B.W. performed movie denoising and motion correction. G.S. and T.N.M. contributed the Mac-1 KO data. J.S. and K.B. wrote the manuscript with the help of L.S. and K.L.

\section{Additional information}

Supplementary Information accompanies this paper at http://www.nature.com/ naturecommunications

Competing financial interests: The authors declare no competing financial interests.

Reprints and permission information is available online at http://npg.nature.com/ reprintsandpermissions/

How to cite this article: Buscher, K. et al. Protection from septic peritonitis by rapid neutrophil recruitment through omental high endothelial venules. Nat. Commun. 7:10828 doi: $10.1038 /$ ncomms10828 (2016).

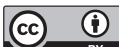

This work is licensed under a Creative Commons Attribution 4.0 International License. The images or other third party material in this article are included in the article's Creative Commons license, unless indicated otherwise in the credit line; if the material is not included under the Creative Commons license, users will need to obtain permission from the license holder to reproduce the material. To view a copy of this license, visit http://creativecommons.org/licenses/by/4.0/ 